\title{
Positioning Study on a Kind Of Improved DV-HOP Algorithm in Wireless Sensor Network
}

\author{
Jianwei $\mathrm{Xu}^{1}$ and Dan Wang ${ }^{2}$ \\ (Zhejiang Industry Polytechnic College Shaoxing Zhejiang 312000 China)

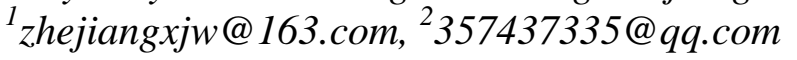

\begin{abstract}
In the WSN, information signal is mainly transmitted through sensors, aiming at the problem that DV- Hop algorithm has low accuracy in node localization, this paper propose establishing the hyperbolic two-dimensional model to determine the distance relationship of the anchor node and unknown node, and then set the error coefficient to reduce the error between the sensor nodes, finally using Stephenson iterative method (Steffensen) positioning method to locate and correct further. Simulation results show that the algorithm in the remote control has high positioning accuracy, so there is certain promotion value for it.
\end{abstract}

Keywords: wireless sensor network; hyperbolic two-dimensional model; error coefficient; Steffensen

\section{Introduction}

With the development of microelectronics technology and computing wireless communication technology, wireless sensor network (wireless sensor network, WSN) gets rapid development. Information transfer among sensors mainly relies on the distribution of node position, so the accurate positioning of a node is the main problem in present study [1].The node localization in WSN mainly refers to the anchor node positioning and unknown node localization, and anchor node refers to a small number of GPS positioning device nodes. The unknown sensor node localization needs anchor nodes to locate. Scholars at home and abroad carried out extensive researches for DV- HOP algorithm and literature [2] proposes that the essence of location problem is to minimize the position error, apply the bat algorithm to optimize the positioning results of DV-HOP. The simulation experiments prove that positioning accuracy is improved, but the complexity of the algorithm is increased; Literature [3] firstly introduces genetic algorithm to calculate the average hop distance of anchor nodes; Then use the average hop distance of the anchor nodes with hop count less than or equal to 3 to weight and process the average hop distance of the unknown nodes. The simulation results show that this algorithm not only improves the accuracy, but also has very good stability; Literature [4] proposes a location algorithm based on weighted three-dimensional DV-Hop. For the calculation of anchor nodes average hop distance, the algorithm sets different weights for anchor nodes with different distances, Build a weighted average hop distance and reduce the positioning error, but the weight set needs further discussion; Literature [5] puts forward the improved algorithm signal strength indicator, and reduces the error by $30 \% \sim 45 \%$; Literature [6] puts forward weighted processing for the hop amount based on the distance between the neighboring nodes, correcting the average hop, narrowing the surrounded delta-shaped area to screen out the final anchor nodes for final calculation; but the algorithm is influenced by other conditions;Literature [7] proposes a general feedback mechanism, introducing the position adjustment factors and on the basis of RSSI hops distance optimization to establish the position information feedback between the unknown node and beacon node location information feedback link. The simulation experiments show that in the network computing environment with changeable resource, the proposed localization 
algorithm can significantly improve the positioning accuracy with more powerful; literature [8] proposes to use the difference between anchor nodes actual distance and the estimated nodes to adjust the average hop value in DV-Hop average algorithm, making the distance error between the anchor nodes and unknown nodes becomes small so that using the least square method recurrence algorithm optimize the position of the nodes. The disadvantage is that the actual environment is difficult to apply.

In order to further improve the positioning accuracy of sensors, the paper introduces Stephenson iteration method based on the DV- HOP algorithm (Steffensen) to modify sensor node localization. Analyzing the disadvantages of DV-Hop algorithm, creating hyperbola two-dimensional equation, reducing the deviation by setting error coefficient and applying Steffensen iteration method to and correct the positioning result of DV- Hop algorithm, the simulation experiment shows that this algorithm can effectively improve the positioning performance.

\section{DV-Hop Algorithm and Error Analysis}

\subsection{DV-Hop Positioning Algorithm}

The detailed steps of DV-Hop algorithm is as following:

(1) Anchor nodes send broadcasting data bag to the surrounding nodes. The unknown nodes will add 1 and send to the next node after receiving the minimum hop after receiving the anchor nodes.

(2) After anchor nodes receiving the information and hop number from other nodes, calculate the distance of each hop for estimation according to formula (1).

$$
\text { HopSize }_{i}=\sum_{j \neq i} \sqrt{\left(x_{i}-x_{j}\right)^{2}+\left(y_{i}-y_{j}\right)^{2}} / \sum_{j \neq i} h o p S_{i j}
$$

In the formula, it's the coordinate of anchor nodes ${ }^{i}$ and ${ }^{j}$, and $h o p S_{i j}$ is the minimum hop between anchor nodes $i$ and ${ }^{j}$

(3) The estimated distance between anchor node and unknown node is:

$$
L_{i}=S_{i} \times \text { HopSize }
$$

According to the distance between the unknown node and each anchor node, use multilateral method to calculate the coordinate of the unknown nodes, as following:

$$
\left\{\begin{array}{c}
\left(x_{i}-x_{1}\right)^{2}+\left(y_{i}-y_{1}\right)^{2}=L_{1}^{2} \\
\left(x_{i}-x_{2}\right)^{2}+\left(y_{i}-y_{2}\right)^{2}=L_{2}^{2} \\
\vdots \\
\left(x_{i}-x_{j}\right)^{2}+\left(y_{i}-y_{j}\right)^{2}=L_{j}^{2}
\end{array}\right.
$$

In the formula, $\left(x_{i}, y_{i}\right)$ is the coordinate of unknown nodes; $\left(x_{1}, y_{1}\right),\left(x_{2}, y_{2}\right), \cdots\left(x_{j}, y_{j}\right)$ are the coordinates of anchor nodes recorded by this unknown node. So through the formula (4), the unknown node $\left(x_{i}, y_{i}\right)$ can get:

$$
P=\left(A^{T} A\right)^{-1} A^{T} B
$$

Set A as:

$$
B=\left[\begin{array}{l}
d_{n}^{2}-d_{1}^{2}+x_{1}^{2}-x_{n}^{2}+y_{1}^{2}-y_{n}^{2} \\
d_{n}^{2}-d_{2}^{2}+x_{2}^{2}-x_{n}^{2}+y_{2}^{2}-y_{n}^{2} \\
\cdots \cdots \cdots \cdots \cdots \cdots \cdots \cdots \cdots \cdots \cdots \\
d_{n}^{2}-d_{i}^{2}+x_{i}^{2}-x_{n}^{2}+y_{i}^{2}-y_{n}^{2}
\end{array}\right]
$$




$$
P=\left[\begin{array}{l}
x \\
y
\end{array}\right]
$$

\subsection{Error Analysis}

In DV-HOP algorithm, the distance between anchor nodes is mainly used to estimate the distance from the unknown node and anchor node. But there are some limits, because it mainly depends on whether the anchor nodes are distributed evenly. But the actual situation is on the contrary, the positioning of anchor nodes is influenced by many factors. When the anchor nodes in the network are distributed densely, the distance between unknown nodes in the network is close to the actual distance. But when the anchor nodes are distributed sparsely, the deviation will be easy to appear, as shown in Figure 1.
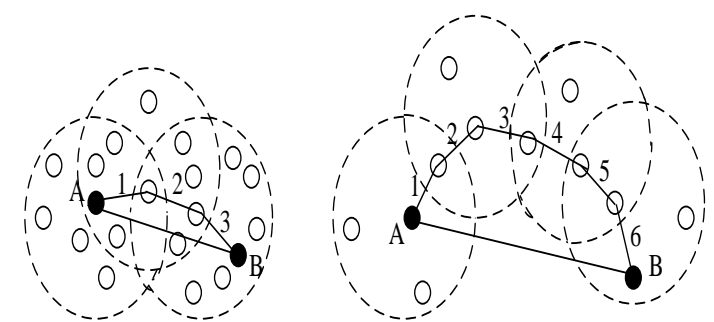

\section{Figure 1. The Error Caused by Nodes Distribution}

Therefore, there must be some error between the distance $L$ measured by DV-Hop algorithm and the actual distance. Assume $f_{n}$ is the distance-measuring error between the unknown node and anchor node, and the distance from the unknown node to the anchor node is shown as formula (5):

$$
\left(x_{i}-x_{j}\right)^{2}+\left(y_{i}-y_{j}\right)^{2}=L_{j}^{2}+f_{j}
$$

According to formula (3), the unknown nodes coordinate can meet the following formula:

$$
\left\{\begin{array}{l}
\left(x_{i}-x_{1}\right)^{2}+\left(y_{i}-y_{1}\right)^{2}=L_{1}^{2}+f_{1} \\
\left(x_{i}-x_{2}\right)^{2}+\left(y_{i}-y_{2}\right)^{2}=L_{2}^{2}+f_{1} \\
\cdots \cdots \cdots \cdots \cdots \cdots \cdots \cdots \cdots \cdots \cdots \cdots \cdots \cdots \cdots \cdots \\
\left(x_{i}-x_{j}\right)^{2}+\left(y_{i}-y_{j}\right)^{2}=L_{j}^{2}+f_{j}
\end{array}\right.
$$

Solve the unknown nodes coordinate $\left(x_{i}, y_{i}\right)$, and

$$
f\left(x_{i}, y_{i}\right)=\sum_{j=1}^{n}\left(\sqrt{\left(x_{i}-x_{j}\right)^{2}+\left(y_{i}-y_{j}\right)^{2}}-L_{j}\right)
$$

When (7) has the minimum value, the overall error is the least and the unknown nodes positioning can be close to the actual value. That is meeting the unknown nodes coordinate of the following formula:

$$
f\left(x_{i}, y_{i}\right)_{\min }=\min \left(\sqrt{\left(x_{i}-x_{j}\right)^{2}+\left(y_{i}-y_{j}\right)^{2}}-L_{j}\right)
$$

Change DV-Hop positioning error problem into non-linear optimization problem through the method aforesaid. Solve through Steffensen iteration method and optimize the sensor positioning accuracy.

\section{Improved DV-Hop Algorithm in WSN}

\subsection{Two-dimension Plane based on the Hyperbola}

To reduce the research complexity, the paper selects $2 \mathrm{D}$ plane as the research object. Assume there are $M$ sensor nodes, the coordinate of the $\mathrm{i}$ unknown node is 
$M_{i, j}=\left[x_{i}, y_{i}\right]^{T}(i=1,2, \cdots n, j=1,2, \ldots \ldots n)$, and the coordinate of the anchor node is $S_{p}=[a, b]^{T}$. So the distance between the origin and the sensor is $\left\|M_{i}-S_{p}\right\|$. Select the $j$ node as reference node and use the measured value $\kappa_{i, j}(i=1, \ldots \ldots . . N)$ to express the incidence relationship between the anchor node and unknown node. Set the relationship between the anchor node and the unknown node is hyperbolic equation, as shown is Figure 2.

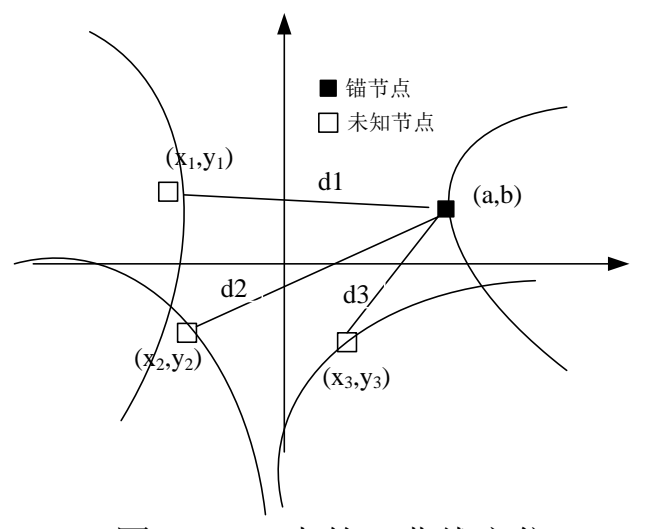

图 $2 \mathrm{WSN}$ 中的双曲线定位

\section{Figure 2. Hyperbola Positioning in WSN}

Set the propagation velocity between signals as $v$, the distance between anchor node and unknown node $d=v \kappa_{i, j}$, construct $\mathrm{N}$ hyperbolic equation $d_{i, j}=d_{i}-d_{j}$ with the anchor node as the center, and get the positioning node coordinate after solving $\mathrm{N}$ equations. Due to the equation in non-linear equation, it should be done linear processing, and expand from $d_{i}^{2}=\left(d_{i, j}+d_{j}\right)^{2}$, as following:

$$
d_{i, j}^{2}+2 d_{i, j} d_{j}+3 d_{i}^{2}=M_{i, j}^{2}+S_{p}-2 S M_{i, j}
$$

Local processing for formula (9) to get formula (10)

$$
M_{i, j}^{2}+S_{p}^{2}-2 M_{, i} S_{\bar{p}}
$$

Substitute formula (10) into (9) to get:

$$
M_{i, j}^{2}-S_{p}^{2}-2\left(M_{i, j}-M_{1,1}\right)^{T} S_{p}=d_{i, j}^{2}+2 d_{i, j} d_{j}
$$

Through formula (11), transform the problem into the solution of linear equation, substitute into $(8)$ for solution.

3.2Error coefficient setting

Because the actual distance between anchor node and the unknown node has difference with the theoretical distance, decreasing through setting the error coefficient, the actual distance between the unknown node and anchor node described previously is $D_{i, j}$,

$$
D_{i, j}=\sqrt{\left(x_{i}-x_{j}\right)^{2}+\left(y_{i}-y_{j}\right)^{2}}
$$

Get the overall error $\varepsilon$ between the unknown node and the anchor node according to formula (12) and (13), such as formula (13), the error coefficient is determined as formula (14):

$$
\begin{gathered}
\varepsilon=\sum_{i \neq j} D_{i j}-L_{i j} \\
f_{i j}=\frac{\varepsilon}{n *(n-1) / 2}
\end{gathered}
$$

In $(14), n *(n-1) / 2$ refers to the hop sum among $\mathrm{n}$ nodes, $f_{i j}$ refers to the error coefficient. 


\subsection{Steffensen Algorithm}

For non-linear equation $f(x)=0$, it can be transformed into the equivalent equation:

$$
x=\varphi(x)
$$

In which, $\varphi(x)$ is called iterated function.

Steffensen iteration method is a kind of improved fixed point iteration method, with advantages of second order rate of convergence, high calculation accuracy, and the iteration function as follows:

$$
\varphi(x)=x-\frac{\left(\varphi(x) x^{2}\right)}{\varphi(\varphi(x))-2 \varphi(x)+x}
$$

Steffensen iterative formula:

$$
\left\{\begin{array}{c}
x_{k}=\varphi\left(x_{k}\right), z_{k}=\varphi\left(y_{k}\right) \\
y_{k+1}=x_{k}-\frac{\left(y_{k}-x_{k}\right)^{2}}{z_{k}-2 y_{k}+x_{k}}, k=0,1,2, \ldots
\end{array}\right.
$$

Steffensen algorithm process as following:

(1) Set the maximum iterations as $\mathrm{N}$, and accuracy as $\varepsilon$, let $k=0$.

(2) Calculate $y_{k}=\varphi\left(x_{k}\right), z_{k}=\varphi\left(y_{k}\right)$,.

(3) If $\left|x_{k+1}-x_{k}\right|<\varepsilon$, stop calculation.

(4) If $k=N$, then stop calculation; unless let $k=k+1$, go to step (2).

\subsection{Steffensen Corrected DV-Hop Positioning Error}

In addition to the setting of error coefficient, the necessary condition for (8) getting the minimum value is:

$$
\frac{\partial f}{\partial x_{i}}=\frac{\partial f}{\partial y_{i}}=0,(m<i \leq n)
$$

In which:

$$
\left\{\begin{array}{l}
\frac{\partial f}{\partial x_{i}}=\sum_{\substack{m<i \leq n \\
1 \leq j \leq m}}\left(2\left(x_{i}-x_{j}\right)-\frac{2 l_{i j}\left(x_{i}-x_{j}\right)}{\sqrt{\left(x_{i}-x_{j}\right)^{2}+\left(y_{i}-y_{j}\right)^{2}}}\right) \\
\frac{\partial f}{\partial y_{i}}=\sum_{\substack{m<i \leq n \\
1 \leq j \leq m}}\left(2\left(y_{i}-y_{j}\right)-\frac{2 l_{i j}\left(y_{i}-y_{j}\right)}{\sqrt{\left(x_{i}-x_{j}\right)^{2}+\left(y_{i}-y_{j}\right)^{2}}}\right)
\end{array}\right.
$$

Let:

$$
\Delta_{i}=\sqrt{\left(\frac{\partial f}{\partial x_{i}}\right)^{2}+\left(\frac{\partial f}{\partial y_{i}}\right)^{2}}
$$

Then the problem for getting the best solution of $\min ($ fitness ()$)$ is transformed to the problem of the unknown node coordinate $\left(x_{i}, y_{i}\right)$ when getting the minimum value of $\Delta_{i}$. The detailed steps as following:

(1) Iterate variables $x_{i}$ and $y_{i}$ according to the first step pf Steffensen iterative formula (17):

$$
\left\{\begin{array}{l}
\bar{x}_{i}=\varphi\left(x_{i}\right)=\frac{\sum_{1 \leq j \leq m}\left(x_{j}+\frac{l_{i j}\left(x_{i}-x_{j}\right)}{\sqrt{\left(x_{i}-x_{j}\right)^{2}+\left(y_{i}-y_{j}\right)^{2}}}\right)}{m} \\
\overline{y_{i}}=\varphi\left(y_{i}\right)=\frac{\sum_{1 \leq j \leq m}\left(y_{j}+\frac{l_{i j}\left(y_{i}-y_{j}\right)}{\sqrt{\left(x_{i}-x_{j}\right)^{2}+\left(y_{i}-y_{j}\right)^{2}}}\right.}{m}
\end{array}\right.
$$




$$
\left\{\begin{array}{c}
\left.\tilde{x}_{i}=\varphi\left(\bar{x}_{i}\right)=\frac{\sum_{1 \leq j \leq m}\left(x_{j}+\frac{l_{i j}\left(x_{i}-x_{j}\right)}{\sqrt{\left(\bar{x}_{i}-x_{j}\right)^{2}+\left(y_{i}-y_{j}\right)^{2}}}\right)}{m}\right) \\
\tilde{y}_{i}=\varphi\left(\bar{y}_{i}\right)=\frac{\sum_{1 \leq j \leq m}\left(y_{j}+\frac{l_{i j}\left(\bar{y}_{i}-y_{j}\right)}{\sqrt{\left(x_{i}-x_{j}\right)^{2}+\left(\bar{y}_{i}-y_{j}\right)^{2}}}\right.}{m}
\end{array}\right.
$$

In the formula, $x_{i}$ and $y_{i}$ are the estimated position of the sensor nodes applying DV-Hop algorithm and take it as the iteration initial value.

(2) Update the iteration for the unknown nodes coordinate according to formula (17)

$$
\left\{\begin{array}{l}
x_{i}^{\prime}=\tilde{x_{i}}-\frac{\left(\tilde{x_{i}}-\bar{x}_{i}\right)^{2}}{\tilde{x}_{i}-2 \overline{x_{i}}+x_{i}} \\
y_{i}^{\prime}=\tilde{y_{i}}-\frac{\left(\tilde{y_{i}}-\overline{y_{i}}\right)^{2}}{\tilde{y_{i}}-2 \bar{y}_{i}+y_{i}}
\end{array}\right.
$$

(3) Substitute the node coordinate $\left(x_{i}, y_{i}\right)$ of the new unknown sensor into formula (20), and get the value of $\Delta_{i}$.

(4) When the iterations reach to the set maximum value, iteration algorithm stops. Get the unknown nodes coordinate that is close to the actual value and minimize the positioning error.

\section{Algorithm Simulation and Analysis}

The hardware for simulation experiment is Core I3 CPU, 4GDDR3 500G hard disk, software using matlab2012. Compare the algorithm with basic DV-HOP algorithm and the algorithm in literature [2]. Select $100 \mathrm{~m} * 100 \mathrm{~m}$ area and 1000 nodes, 220 for anchor nodes, 780 for unknown nodes, and all the nodes are distributed randomly. Using error calculation formula (24) as the comparison standard, and $\left(x_{i}^{\prime}, y_{i}^{\prime}\right)$ and $\left(x_{i}, y_{i}\right)$ are used to refer to the estimated unknown and actual unknown, $R$ is the node communication radius, $N$ is the node number. The results as following:

$$
f=\frac{\sum_{i \neq j} \sqrt{\left(x_{i}^{\prime}-x_{i}\right)^{2}+\left(y_{i}^{\prime}-y_{i}\right)^{2}}}{R \square N}
$$

Figure 3 shows the relationship between different nodes density and positioning error. From the figure, we can see that as the nodes density increases, the positioning error is optimized, from which, we can find that the error generated from the algorithm in this paper is the least. Comparing with DV-HOP algorithm, it optimizes $21.2 \%$, and $7.23 \%$ comparing with literature [2], which can indicates that the algorithm in this paper can get more accurate positioning results. 


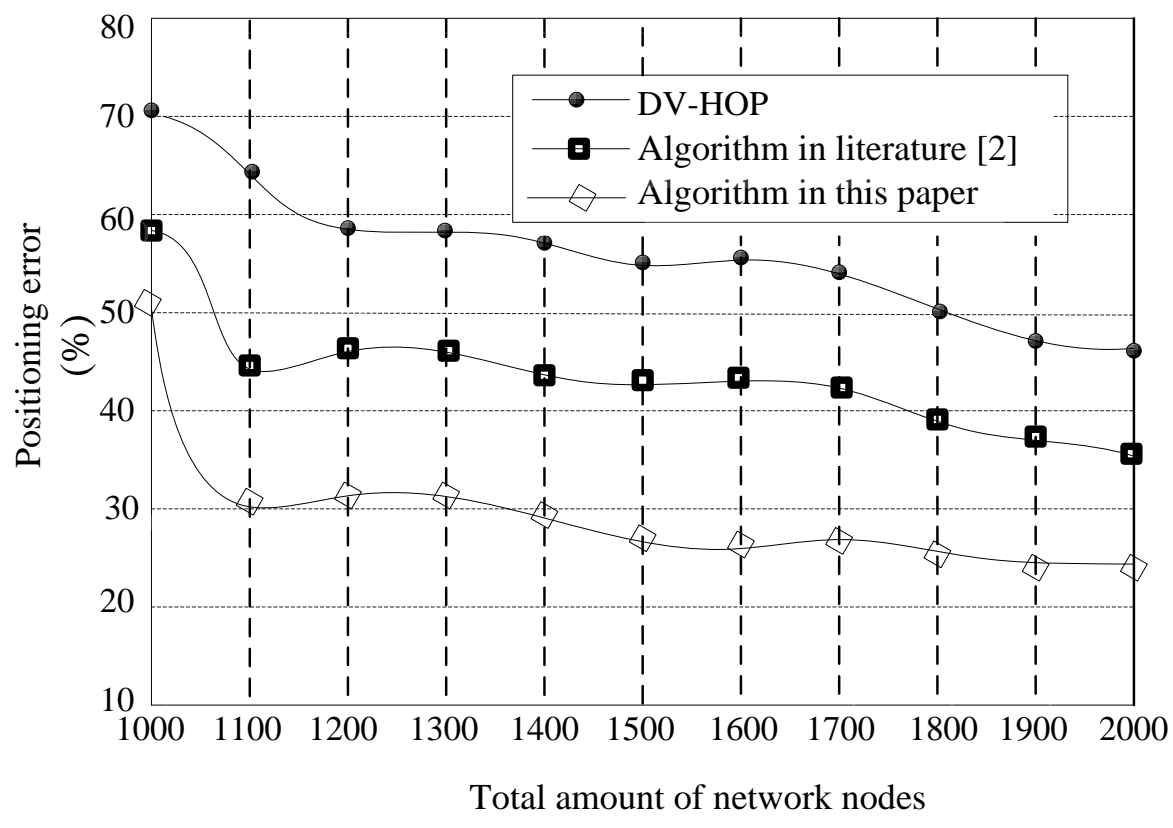

Figure 3. The Effect of Node Density for Positioning Error

Figure 4 shows the relationship between different node communication radius and positioning error. Nodes communication radius concerns the accuracy of the unknown nodes. Select the node radius from 5 to $15 \mathrm{~m}$. From the figure, we can find that the positioning error of these three algorithms decrease with node communication radius increasing, which indicates that all three algorithms improve the relationship with nodes communication radius. The curve of the algorithm in this paper is gentle, indicating the increasing of nodes radius will not affect the stability of the algorithm. Therefore, comparing with other two algorithms, the positioning error in this paper can finish the positioning more accurately.

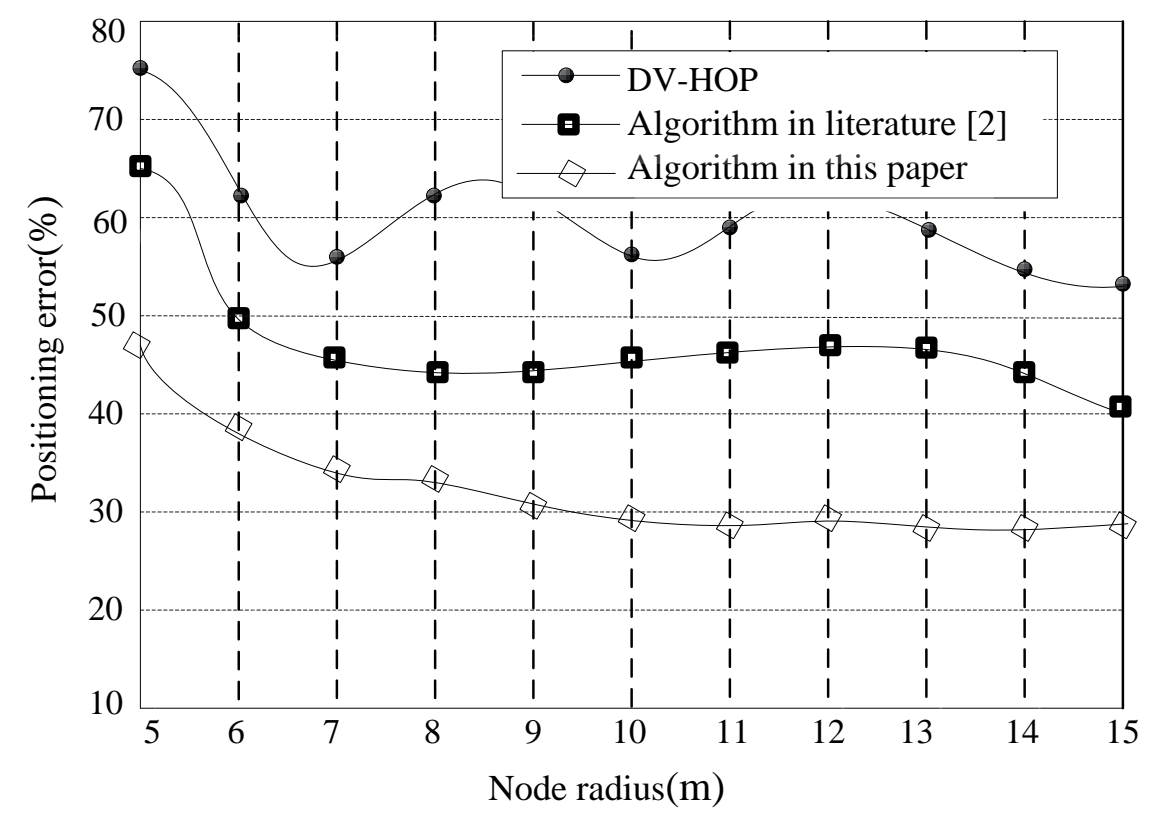

Figure 4. The Effect for Poisoning Error by Nodes Communication Radius

Figure 5 shows the relationship between anchor nodes proportion and positioning error. The proportion of anchor nodes in three algorithms increases from $2 \%$ to $20 \%$. From the figure, we can find that comparing with the other two algorithms under the condition of 
increasing anchor node proportion, positioning error of the algorithm in this paper decreases $12.3 \%$ than DV-HOP and 5.2\% than the algorithm in literature [2], which indicates that with the anchor nodes increasing, the algorithm in this paper has more obvious positioning advantages.

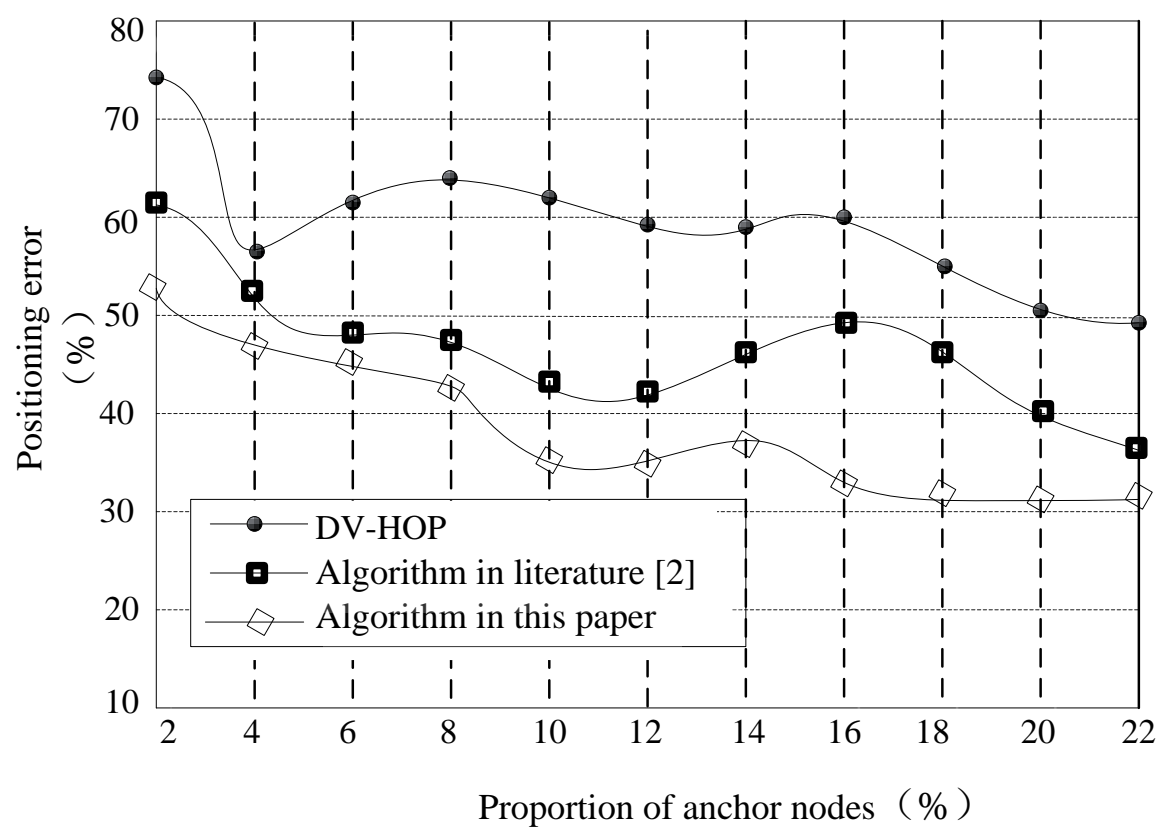

Figure 5. The Effect for Positioning Error of Anchor Nodes Proportion

\section{Conclusion}

For the DV-Hop positioning issues in WSN, based on the DV-Hop algorithm, the paper introduces hyperbola 2D plane equation to position the relationship between the anchor nodes and the unknown nodes. Secondly, decrease the distance error by introducing the error positioning coefficient and apply Steffensen iteration method to analyze and correct the positioning results to realize accurate positioning. Through the comparison of three algorithms in the simulation experiment, the algorithm in this paper can increase the positioning accuracy effectively, so it is practical to some extent.

\section{References}

[1] Huang yi,Hu ai-qun.Survey on Localization Algorithms for Wireless Sensor Networks[J].Telecommunications science,2010,20(7):69-74

[2] Cao yu-xiao,Zhang ian.Improvement of DV-Hop Localization Based on Bat Algrothrim[J].Computer Measurement \& Control,2015,23(4):1273-1275

[3] Zhang wan-li.An improved DV-Hop algorithm based on genetic algorithm[J].Journal of Chongqing University (Natural Science Edition),2015,38(3):159-166

[4] Li lin.A Weighted 3D DV-Hop Localization Algorithm[J].Control Engineering of China,2015,22(4):761-764

[5] Wu chun-ming,Zhang jin-long.Improved DV-HOP positioning algorithm using continuous hop count based on RSSI[J]. Journal of Chongqing University of Posts and Telecommunications(Natural Science Edition),2015,27(2):184-187

[6] Cai lin-ping,Jiang jin-jin.An Improved DV-HOP Localization Algorithm[J].Microelectronics \& Computer,2015,32(4):120-124

[7] Liu fei.Improved DV-hop localization algorithm based on RSSI and feedback mechanism[J].Journal of Nanjing University of Posts and Telecommunications(Natural Science),2015,35(2):108-113

[8] Su bin,Xue wei-jie,Wang hong-yuan.An Error Improved Method Based on Range-free Localization DV-Hop Algorithm in WSN[J],Computer Measurement \& Control,2013,21(5):1357-1359 


\section{Authors}

Jianwei Xu, (1980- ), Male, Master's Degree, Research Direction: intelligent control, embedded system technology, detection device and instrumentation.

Dan Wang, (1978- ), Female, Master's Degree, Research Direction: mechanical and electrical control and automation, dynamic testing and fault diagnosis of mechanical system. 
International Journal of Grid and Distributed Computing Vol. 9, No. 8 (2016) 